\title{
Numerical Study of Pressure Fluctuations due to a Mach 6 Turbulent Boundary Layer
}

\author{
Lian Duan* \\ National Institute of Aerospace, Hampton, VA 23666 \\ Meelan M. Choudhari ${ }^{\dagger}$ \\ NASA Langley Research Center, Hampton, VA 23681
}

\begin{abstract}
Direct numerical simulations (DNS) are used to examine the pressure fluctuations generated by a Mach 6 turbulent boundary layer with nominal freestream Mach number of 6 and Reynolds number of $R e_{\tau} \approx 464$. The emphasis is on comparing the primarily vortical pressure signal at the wall with the acoustic freestream signal under higher Mach number conditions. Moreover, the Mach-number dependence of pressure signals is demonstrated by comparing the current results with those of a supersonic boundary layer at Mach 2.5 and $R e_{\tau} \approx 510$. It is found that the freestream pressure intensity exhibits a strong Mach number dependence, irrespective of whether it is normalized by the mean wall shear stress or by the mean pressure, with the normalized fluctuation amplitude being significantly larger for the Mach 6 case. Spectral analysis shows that both the wall and freestream pressure fluctuations of the Mach 6 boundary layer have enhanced energy content at high frequencies, with the peak of the premultiplied frequency spectrum of freestream pressure fluctuations being at a frequency of $\omega \delta / U_{\infty} \approx 3.1$, which is more than twice the corresponding frequency in the Mach 2.5 case. The space-time correlations indicate that the pressure-carrying eddies for the higher Mach number case are of smaller size, less elongated in the spanwise direction, and convect with higher convection speeds relative to the Mach 2.5 case. The demonstrated Mach-number dependence of the pressure field, including radiation intensity, directionality, and convection speed, is consistent with the trend exhibited in experimental data and can be qualitatively explained by the notion of 'eddy Mach wave' radiation.
\end{abstract}

\section{Nomenclature}

$C_{p} \quad$ heat capacity at constant pressure, $\mathrm{J} /(\mathrm{K} \cdot \mathrm{kg})$

$C_{p p}$ Space-time correlation coefficient of the pressure field, dimensionless

$C_{v} \quad$ heat capacity at constant volume, $\mathrm{J} /(\mathrm{K} \cdot \mathrm{kg})$

$H$ shape factor, $H=\delta^{*} / \theta$, dimensionless

$M \quad$ Mach number, dimensionless

$M_{r} \quad$ relative Mach number, $M_{r}=\left(U_{\infty}-U_{s}\right) / a_{\infty}$, dimensionless

$\operatorname{Pr}$ Prandtl number, $\operatorname{Pr}=0.71$, dimensionless

$R \quad$ ideal gas constant, $R=287, \mathrm{~J} /(\mathrm{K} \cdot \mathrm{kg})$

$R e_{\theta}$ Reynolds number based on momentum thickness and freestream viscosity, $R e_{\theta} \equiv \frac{\rho_{\infty} U_{\infty} \theta}{\mu_{\infty}}$, dimensionless

$R e_{\delta 2}$ Reynolds number based on momentum thickness and wall viscosity, $R e_{\delta_{2}} \equiv \frac{\rho_{\infty} U_{\infty} \theta}{\mu_{w}}$, dimensionless

$R e_{\tau}$ Reynolds number based on shear velocity and wall viscosity, $R e_{\tau} \equiv \frac{\rho_{w} u_{\tau} \delta}{\mu_{w}}$, dimensionless

$T$ temperature, $\mathrm{K}$

$T_{r} \quad$ recovery temperature, $T_{r}=T_{\infty}\left(1+0.9 * \frac{\gamma-1}{2} M_{\infty}^{2}\right), \mathrm{K}$

$U_{b} \quad$ bulk convection speed of pressure-carrying eddies, $\mathrm{m} / \mathrm{s}$

$U_{s} \quad$ convection speed of 'effective' radiation sources, $\mathrm{m} / \mathrm{s}$

* Research Scientist. Member, AIAA

${ }^{\dagger}$ Aerospace Technologist, Computational AeroSciences Branch, M.S. 128. Associate Fellow, AIAA 


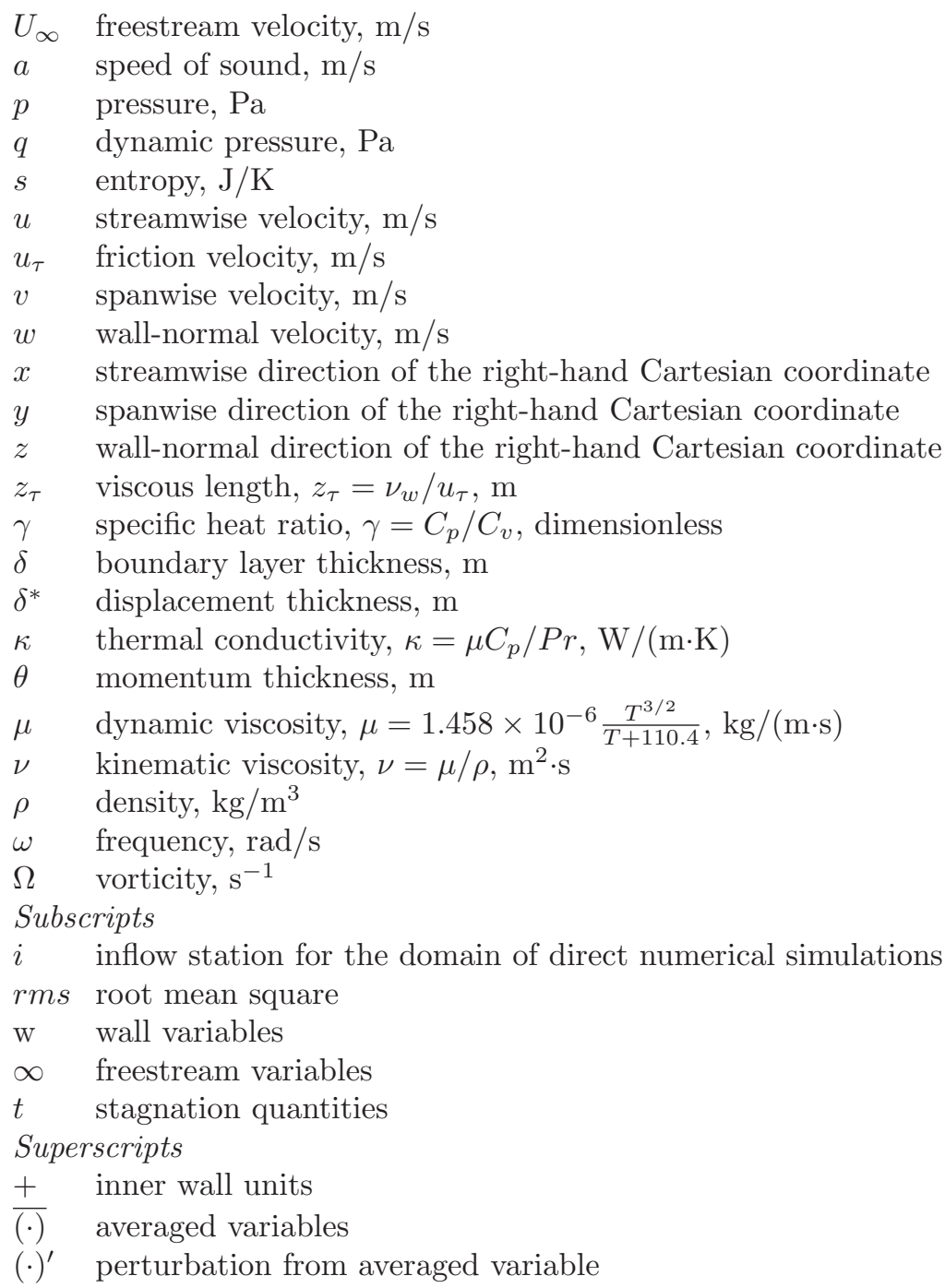

\section{Introduction}

It is well known that the free-stream disturbances in a conventional high-speed facility are typically dominated by acoustic radiation from the turbulent tunnel wall boundary layers. ${ }^{1-13}$ The elevated freestream disturbance levels in conventional (i.e., noisy) high-speed wind tunnels usually result in an earlier onset of transition relative to that in a flight environment or in a quiet tunnel. Yet, the conventional facilities continue to be used for transition sensitive measurements because of the size and Reynolds number limitations of existing quiet facilities and the prohibitive cost of flight tests. To enable a better use of transition data from the conventional facilities, it is important to understand the acoustic fluctuation field that dominates the freestream disturbance environment in those facilities. Measurements at Purdue University indicate that the effect of tunnel disturbances on transition cannot be quantified in terms of a single metric corresponding to the root-mean-square amplitude of freestream acoustic disturbances. ${ }^{14}$ With increased knowledge of the receptivity mechanisms of high-speed boundary layers, ${ }^{15,}{ }^{16}$ it becomes particularly important to characterize the details of the tunnel acoustics originating from the tunnel-wall turbulent boundary layers. Even during flight, the laminar boundary layer flow over the aerodynamic surface of interest may be exposed to acoustic radiation from the turbulent boundary layer over other neighboring surfaces, not unlike a test model mounted within a conventional wind tunnel.

With the exception of early measurements by Laufer ${ }^{1}$ and a few others, ${ }^{5}$ all previous studies of pressure fluctuations due to high-speed turbulent boundary layers have focused on the surface pressure fluctuations underneath the boundary layer, and the existing data exhibit a considerable degree of scatter. ${ }^{17-19}$ As pointed out by several authors, ${ }^{19,20}$ there are few (if any) reliable measurements of the variance of the wall 
pressure fluctuations and its frequency spectra at high speeds, due to either a poor spatial resolution of the pressure transducers and/or the limited frequency response of these sensors. Laufer's measurements of the acoustic fluctuations in the freestream region ${ }^{1}$ are also subject to analogous sources of experimental error. Moreover, as noted by Laufer, the interpretation of disturbance measurements in a wind tunnel is further complicated by the fact that the measurements reflect the combined outcome of acoustic radiation from all sides of the tunnel wall. As a result, highly accurate measurements of the absolute amplitudes of the radiated acoustic energy were not pursued during his experiments and only the statistical quantities that were least likely to be influenced by the presence of multiple tunnel walls were investigated.

Direct numerical simulations (DNS) can overcome many of the aforementioned difficulties with experimental measurements of freestream disturbances as well as provide access to quantities that cannot be measured easily. The DNS can also isolate freestream disturbances due to acoustic radiation, thereby avoiding any contamination due to secondary sources such as vortical and entropy fluctuations in the incoming stream. The successful application of DNS for capturing the freestream acoustic pressure fluctuations has been demonstrated by the current authors in the context of a simulation of a Mach 2.5 supersonic turbulent boundary layer, in which many single and multi-point statistics of the pressure field, including the pressure intensities, frequency spectra, space-time correlations, and convection speeds, were described. ${ }^{21}$ The current study extends that previous study into the hypersonic Mach number regime (where even the properties of surface pressure fluctuations have not been quantified as yet) to highlight the variation of acoustic characteristics with Mach number as well as to provide additional validation of simulations against experimentally measured trends.

The flow conditions selected for the numerical simulation of a hypersonic turbulent boundary layer and the numerical method used for this simulation are outlined in Section II. Section III is focused on an analysis of the statistical characteristics of freestream pressure fluctuations, including power spectral densities, pressure fluctuation intensities, integral length scales, and convection speeds. Conclusions thus far are outlined in Section IV.

\section{Flow conditions and numerical methodology}

Table 1 outlines the flow conditions for the Mach 6 simulation. These conditions are similar to the operation conditions of Purdue Mach 6 Quiet Tunnel. Flow conditions for the previous DNS simulation of Mach 2.5 boundary layer are also included to facilitate the comparison between different Mach number cases. Observe that the values of $R e_{\tau}$ and $R e_{\delta 2}$ are similar for both DNS cases, allowing a meaningful comparison between the two simulations. $T_{w} / T_{r}$ is approximately 0.8 for the Mach 6 simulation and 1 for the Mach 2.5 simulation.

Figure 1 shows the general computational set-up for the DNS in the present work, which parallels the setup in Duan et al. ${ }^{21}$ for the Mach 2.5 simulation, wherein the effects of domain size and grid resolution were also assessed. The choice of grid parameters for the present study is based on lessons learned from Duan et al., ${ }^{21}$ allowing a single simulation to be used to assess the effects of Mach number. The domain size for the present computation is $\left(58.7 \delta_{i}, 15.7 \delta_{i}, 39.7 \delta_{i}\right)$ in the stream-wise, span-wise, and wall-normal directions, respectively. The streamwise domain length, $L_{x}=58.7 \delta_{i}$, is selected to be larger than the eddy decorrelation distance to guarantee minimal spurious correlation being introduced due to the inflow turbulence generation. The number of grid points is $(1600,800,500)$ in the streamwise, spanwise, and wallnormal directions, respectively. Uniform grid spacings are used in the streamwise and spanwise directions with grid spacings $\Delta x^{+}=10.2$ and $\Delta y^{+}=5.5$, respectively. The grids in the wall-normal direction are clustered in the boundary layer with $\Delta z^{+}=0.55$ at the wall, and kept uniform with $\Delta z^{+}=5.7$ in the freestream until up to approximately $6 \delta_{i}$. Such grids are designed to adequately resolve both the boundary layer and the near field of acoustic fluctuations radiated by the boundary layer. Unless otherwise stated, the grid resolutions specified in this section are normalized by the viscous length scale $z_{\tau}$ at the inflow plane. The computational grid resolution inside the boundary layer is comparable to those reported in the literature in the context of previous simulations of turbulent wall-bounded flows using comparable numerical algorithms..$^{22-25}$

The details of the DNS methodology, including numerical methods, initial and boundary conditions, has been documented in our previous paper. ${ }^{21}$ Therefore, only a cursory description is given here. The full three-dimensional compressible Navier-Stokes equations in conservation form are solved in generalized

curvilinear coordinates. The working fluid is assumed to be a perfect gas and the usual constitutive relations 
for a Newtonian fluid are used: the viscous stress tensor is linearly related to the rate-of-strain tensor, and the heat flux vector is linearly related to the temperature gradient through the Fourier's law. A 7th-order weighted essentially non-oscillatory (WENO) scheme 26,27 is used to compute the convective flux terms. For the viscous flux terms, a 4th order central difference scheme is used. The 3rd order low storage Runge-Kutta scheme by Williamson ${ }^{28}$ is used for time integration.

The turbulent inflow is generated using the recycling/rescaling method developed by $\mathrm{Xu}$ and $\mathrm{Martin}^{29}$ with the recycling station set at $56.7 \delta_{i}$ downstream of the inlet to allow for adequate decorrelation between the recycling station and the inflow. Dynamical translation operations ${ }^{30}$ are applied to the recycled turbulence plane at randomly-distributed time intervals to improve the low-frequency characteristics of recycling/rescaling inflow turbulence generation. On the wall, no-slip conditions are applied for the three velocity components and an isothermal condition is used for the temperature with $T_{w} \approx 0.8 T_{r}$. At the

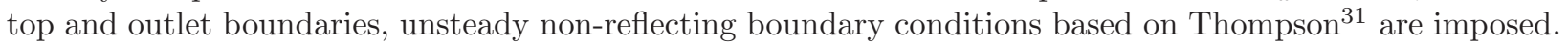
Periodic boundary conditions are used in the spanwise direction.

For the current spatial simulation, the boundary layer grows slowly in the streamwise direction, with corresponding variations in $\delta$ from $13.8 \mathrm{~mm}$ at the inlet to $24.5 \mathrm{~mm}$ near the exit and $R e_{\theta}$ from 5400 to 9750. The stremwise computational domain is large enough for the memory of the inflow generation to fade out and a nearly uniform acoustic radiation field to be established. In particular, Figure 2 shows that the pressure fluctuations at the wall and in the freestream have become nearly homogeneous in the streamwise direction after $x / \delta_{i} \approx 35$.

In the following section, averages are first calculated over a streamwise window of $\left[x_{a}-0.9 \delta_{i}, x_{a}+0.9 \delta_{i}\right]$, $x_{a}=55 \delta_{i}$, and spanwise locations for each instantaneous flow field; then, an ensemble average over flow field snapshots spanning a time interval of approximately $240 \delta_{i} / U_{\infty}$ is calculated. To monitor the statistical convergence, flow statistics are computed by averaging over the whole or half the number of the flow-field snapshots and negligible difference $(<1 \%)$ is observed between the two.

Power spectra are calculated using the Welch method ${ }^{32}$ with 8 segments and $50 \%$ overlap. A Hamming window is used for weighting the data prior to the fast Fourier transform (FFT) processing. The sampling frequency is approximately $63 U_{\infty} / \delta_{i}$, or $4 \mathrm{MHz}$, and the length of an individual segment is approximately $53.2 \delta_{i} / U_{\infty}$. Negligible difference in spectral estimation is observed within the reported frequency range, when the overall time record is subdivided in 12 segments instead of the baseline number of 8 segments. In addition, the DNS methodology has been extensively validated in previous work for supersonic/hypersonic turbulent boundary layers ${ }^{22-25}$ and for supersonic shock wave-turbulent boundary layer interactions. ${ }^{33-35}$

\section{Statistical and spectral analysis}

The main parameters of the boundary layer at the station selected for the statistical analysis $\left(x_{a}=55 \delta_{i}\right)$ are listed in Table 1. The van-Driest transformed mean velocity profile from the DNS is shown in Figure 3. The mean velocity conforms well to the incompressible law-of-the-wall and shows a logarithmic region upon van Driest transformation.

\section{A. Characteristics of freestream fluctuations}

In this section, the characteristics of freestream fluctuations are analyzed using the theory of modal analysis, which was initially proposed by Kovasznay ${ }^{36}$ and further developed by Logan ${ }^{37}$ and Smits \& Dussauge. ${ }^{38}$ According to Kovasznay, ${ }^{36}$ the fluctuations at any point are represented as a superposition of three modes: the sound-wave mode (also referred to as the acoustic mode), the entropy mode (also called the entropy spottiness or temperature spottiness mode), and the vorticity mode. Modal analysis has been successfully applied by Donaldson \& Coulter ${ }^{9}$ to evaluate the level and the sources of disturbances in AEDC Tunnels A and $\mathrm{B}$, and by Masutti et al. ${ }^{13}$ to characterize the freestream fluctuations in the Mach 6 Hypersonic Wind Tunnel H3 of the von Karman Institute.

Tables 2 and 3 list the freestream values of several fluctuating flow variables for the Mach 6 and 2.5 DNS, respectively. The normalized velocity fluctuations at Mach 6 are larger than those at Mach 2.5. Yet, the rms fluctuations in either velocity component are less than approximately $0.2 \%$. Therefore, it is conceivable that the dynamics of acoustic fluctuations in the freestream is still linear in nature. However, further work is necessary to establish the linearity of the acoustic field. The fluctuations in thermodynamic variables are stronger than the velocity fluctuations and also increases from Mach 2.5 to Mach 6. At Mach 6, the 
rms pressure fluctuations are approximately $2 \%$ of the mean pressure value. Moreover, it is shown that $s_{r m s}^{\prime} / R<<p_{r m s}^{\prime} / \bar{p}$, and by using the values listed in Table 2 , the following isentropic relations are satisfied

$$
\begin{aligned}
\frac{T_{r m s}^{\prime}}{\bar{T}} & \approx \frac{\gamma-1}{\gamma} \frac{p_{r m s}^{\prime}}{\bar{p}} \\
\frac{\rho_{r m s}^{\prime}}{\bar{\rho}} & \approx \frac{1}{\gamma} \frac{p_{r m s}^{\prime}}{\bar{p}}
\end{aligned}
$$

indicating that the freestream fluctuations are nearly isentropic and the contribution from the entropy mode is minimal. The relative importance of acoustic mode and vorticity mode in the freestream can be demonstrated by the ratio of dilatation variance $\overline{\left(\partial u_{i} / \partial x_{i}\right)^{\prime 2}}$ and vorticity variance $\overline{\Omega_{i}^{\prime} \Omega_{i}^{\prime}}$, which are representative of the acoustic and vorticity mode, respectively. The large value of $\overline{\left(\partial u_{i} / \partial x_{i}\right)^{\prime 2}} / \overline{\Omega_{i}^{\prime} \Omega_{i}^{\prime}}$ implies the dominance of acoustic mode over the vorticity mode in the freestream.

The dominance of the acoustic mode over the other two modes confirms that a purely acoustic field in the freestream is successfully isolated by the present DNS. In typical high-speed (noisy) wind tunnels, however, multiple disturbance sources exist and all three modes contribute to the freestream fluctuations. ${ }^{9,13}$ The present simulation thus provides a unique opportunity to study the generic spectral features and production mechanisms particular to the acoustic disturbance.

\section{B. Intensity of pressure fluctuations}

Figure 4a shows that the wall pressure rms normalized by the local wall shear for the Mach 6 case is about 2.8, which is close to the corresponding value of approximately 2.6 at Mach $2.5 .^{21}$ The normalized value is also close to the value $p_{w, r m s}^{\prime} / \tau_{w}=3$ based on the model by Bies ${ }^{39}$ and those given by the DNS of Guarini et al. ${ }^{40}$ Spalart, ${ }^{41}$ and Bernardini \& Pirozzoli ${ }^{42}$ at lower Mach numbers. Outside the boundary layer, $p_{r m s}^{\prime} / \tau_{w}$ approaches a constant value of about 0.9 for the Mach 6 case, which is significantly larger than the Mach 2.5 case with $p_{r m s, \infty}^{\prime} / \tau_{w} \approx 0.4$. The variation of freestream value with Mach number is consistent with the trend predicted by the experimental data reported by Laufer $^{1}$ (Fig. 5).

Figure $4 \mathrm{~b}$ shows the rms pressure profile normalized by the freestream dynamic pressure $\left(q_{\infty}=1 / 2 \rho_{\infty} U_{\infty}^{2}=\right.$ $\left.1 / 2 \gamma p_{\infty} M_{\infty}^{2}\right)$. While $p_{r m s}^{\prime} / q_{\infty}$ shows a strong dependence on Mach number within the boundary layer, it nearly collapses the data in the freestream value with $p_{r m s, \infty}^{\prime} / q_{\infty} \approx 0.25 \%$, suggesting the potential scaling of $p_{r m s, \infty}^{\prime} / \bar{p}_{\infty} \propto M_{\infty}^{2}$ over the Mach number range covered by these two simulations. However, additional data points are necessary to establish the validity of such simple scaling.

\section{Frequency spectra}

The frequency spectrum of the pressure fluctuations is defined as

$$
\Phi_{p}(\omega)=\frac{1}{2 \pi} \int_{-\infty}^{\infty} \overline{p^{\prime}(x, y, z, t) p^{\prime}(x, y, z, t+\tau)} e^{-i \omega \tau} d \tau
$$

Figures $6 \mathrm{a}$ and $6 \mathrm{~b}$ show the pressure spectrum at the wall and in the freestream, respectively. The pressure spectrum is normalized so that the area under each curve is equal to unity. For reference, straight lines with slopes of $2,-1,-7 / 3$, and -6 are also included to gauge the rate of spectral roll-off across relatively low, mid, and high frequencies, respectively. The wall-pressure spectrum for both Mach numbers varies rather weakly with frequency as $\omega \rightarrow 0$. The absence of the more rapid and incompressible $\omega^{2}$ scaling at low frequencies in the wall-pressure spectrum is consistent with the measurements by Beresh et al. ${ }^{19}$ and the DNS by Bernardini \& Pirozzoli ${ }^{42}$ at supersonic Mach numbers. At high frequencies, the wall spectrum for the Mach 6 case has significantly higher energy than the Mach 2.5 case, and the spectrum for both cases exhibits a slightly more rapid decay than the $\omega^{-5}$ scaling predicted theoretically by Blake. ${ }^{43}$ Unlike the Mach 2.5 case, the wall-pressure spectrum for the Mach 6 case shows an apparent $\omega^{-n}$ behavior (with $n \approx 0.7-1$ ) corresponding to a universal overlap region. ${ }^{44}$ Similar behavior is not obvious for the Mach 2.5 case and the DNS results $\left(M_{\infty}=2,3\right.$, and 4$)$ reported by Bernardini \& Pirozzoli. ${ }^{42}$

Similar to the wall pressure, the freestream pressure fluctuations for both Mach number cases weakly decay as $\omega \rightarrow 0$ and exhibit an approximately $\omega^{-5}$ roll-off at high frequencies. The freestream spectrum for the Mach 6 case has significantly higher energy than that for the lower Mach number case at $\omega \delta / U_{\infty}>3$. 
While the freestream spectrum for the Mach 2.5 DNS and the experiments by Masutti et al. ${ }^{13}$ has an observable region of of slope close to $-7 / 3$, a similar region is less evident for the present Mach 6 DNS.

To illustrate the distribution of energy among various frequencies, Fig. 7a shows the pre-multiplied pressure spectra at selected heights above the surface. It is shown that the pressure spectra in the inner layer (including the wall, buffer layer, and log layer) have a dominant hump centered on $\omega \delta / U_{\infty} \approx 8$ (or $f \delta / U_{\infty} \approx$ 1 ), which is the characteristic frequency of the energetic vortical structures within the boundary layer. As one moves away from the wall into the outer layer, the peak gradually shifts to lower frequencies as spatial intermittency becomes more important. In the freestream, where the the pressure signal is predominantly acoustic, the peak of the spectrum is centered at a frequency of $\omega \delta / U_{\infty} \approx 3$ (i.e. $f \approx 108 \mathrm{KHz}$ ), indicating that the characteristic frequency of the acoustic mode is significantly lower than that of the vorticity mode. Similar variation in pre-multiplied pressure spectrum with wall-normal distance is observed for the Mach 2.5 case. Figure $7 \mathrm{~b}$ further compares the pre-multiplied spectra for the two Mach number cases at the wall and in the freestream. While the wall spectrum is centered on nearly the same frequency $\omega \delta / U_{\infty} \approx 8$ at both Mach numbers, the freestream spectrum for the Mach 6 case peaks at significantly higher frequency than the Mach 2.5 case. The reduced gap in the dominant frequency between pressure signals at the wall and in the freestream can be explained by the 'eddy Mach wave radiation' concept ${ }^{45,46}$ as discussed in Subsection D.

\section{Space-time correlations}

The statistical properties of the pressure field are investigated through the space-time correlation coefficient defined as

$$
C_{p p}(\Delta x, \Delta y, \Delta t)=\frac{\overline{p^{\prime}(x, y, z, t) p^{\prime}(x+\Delta x, y+\Delta y, z, t+\Delta t)}}{\left(\overline{p^{\prime 2}(x, y, z, t)}\right)^{1 / 2}\left(\overline{p^{\prime 2}(x+\Delta x, y+\Delta y, z, t+\Delta t)}\right)^{1 / 2}}
$$

where $\Delta x$ and $\Delta y$ are spatial separations in the streamwise and spanwise directions, respectively, and $\Delta t$ is the time delay.

Figures $8 \mathrm{a}$ and $8 \mathrm{~b}$ display the contours of streamwise-spanwise correlation $C_{p p}(\Delta x, \Delta y, 0)$ of the pressure fluctuations at the wall and in the freestream, respectively, for the Mach 6 case. The contours of $C_{p p}(\Delta x, \Delta y, 0)$ for the Mach $2.5 \mathrm{DNS}^{21}$ (dashed lines) are also included to highlight the Mach number dependence. At both heights, the contours are approximately circular for small spatial separation but become elongated in the spanwise direction for large separation distances, indicating that the small-scale pressurecarrying eddies are nearly isotropic while the large-scale eddies become more coherent in the spanwise direction. Relative to the wall pressure fluctuations, which are primarily vortical, the acoustic radiation in the freestream has a significantly greater extent in both in-plane directions, indicating a shift in characteristic length scales between those pressure signals. At the wall, the contours exhibit minor Mach number dependence when nondimensionalized by the boundary layer thickness. In the freestream, however, the large-scale pressure-carrying eddies for the Mach 6 case become less coherent and more isotropic compared to the lower Mach number case.

The space-time correlation contours $C_{p p}(\Delta x, 0, \Delta t)$ of the surface and freestream pressure fluctuations are shown in Fig. 9a and Fig. 9b, respectively. The shape of the contours at both locations indicates the convective nature of the pressure field, which is characterized by downstream propagation of coherent pressure-carrying eddies. The overall larger inclination of the space-time correlation contours for the Mach 6 case indicates the pressure-carrying eddies propagate with a larger convective velocity relative to the Mach 2.5 case, at least at low to intermediate frequencies.

From the standpoint of transition analysis, the convection velocity is an important characteristic of the stochastic acoustic field in the freestream, since the receptivity characteristics are known to be sensitive to the orientation of the plane wave disturbance. ${ }^{15}$ To further demonstrate the convective nature of the pressure signal as well as its scale dependence, the convection speed is computed using the space-time correlation coefficient $C_{p p}(\Delta x, 0, \Delta t)$ following a similar procedure as Bernadini and Pirozzoli. ${ }^{42}$ For a given time delay $\Delta t$ (corresponding to a frequency $\omega=2 \pi / \Delta t$ ), the convection speed $U_{c}$ is defined as the ratio $\Delta x_{1} / \Delta t$ taken at the value of $\Delta x_{1}$ where a local maximum of space-time correlation coefficient is attained. Figure 10a plots the convection speed as a function of frequency at several selected heights for the Mach 6 DNS. The convection velocity shows a frequency dependence, especially close to the wall. At the wall $(z=0)$, in the viscous sublayer $\left(z^{+}=5\right)$, and in the buffer layer $\left(z^{+}=17\right)$, the convection speed is $U_{c} \approx 0.8 U_{\infty}$ for the large-scale disturbances (associated with lower frequencies) and $U_{c} \approx 0.7 U_{\infty}$ for the small-scale disturbances (associated with higher frequencies), which is significantly larger than the local mean velocity. The values 
of convection speed at the wall are consistent with those widely quoted for low-speed flows ${ }^{47-49}$ and those reported by Bernadini and Pirozzoli ${ }^{42}$ for turbulent boundary layers at Mach 2, 3, and 4 . In the $\log \operatorname{layer}$ $(z / \delta=0.16)$ and the outer layer $(z / \delta=0.75)$ of the boundary layer, the convection velocity becomes less frequency-dependent and is nearly equal to the local mean velocity value. In the freestream $(z / \delta=3.2)$, the pressure-carrying eddies propagate at significantly smaller speeds with $U_{c} \approx 0.63 U_{\infty}$. A similar variation of convection speed with the wall-normal distance is observed in the Mach 2.5 case. $^{21}$ In addition, Fig. 10b further compares the convection speeds at the wall and in the freestream between the Mach 6 and Mach 2.5 cases. It is seen that the convection speed both at the wall and in the freestream is larger for the Mach 6 case, consistent with Fig. 9a and Fig. 9b.

To represent the overall convection speed of the pressure-carrying eddies, we define the bulk convection speed $\left(U_{b}\right)$ as that associated with the frequency where the pre-multiplied pressure spectra (Fig. 7a) attain their maximum. The bulk convection speed for the Mach 6 case is $U_{b} \approx 0.71 U_{\infty}$ at the wall and $U_{b} \approx 0.63 U_{\infty}$ in the freestream, compared with $U_{b} \approx 0.66 U_{\infty}$ at the wall and $U_{b} \approx 0.4 U_{\infty}$ in the freestream for the Mach 2.5 case. Figures $11 \mathrm{a}$ and $11 \mathrm{~b}$ show the variation of bulk convection speed with freestream Mach number at the wall and in the freestream, respectively. While the data by Kistler \& Chen ${ }^{17}$ shows that the convection speed at the wall plateaus for $M_{\infty}>2$, the current DNS and the data reported by Bernadini and Pirozzoli ${ }^{42}$ show a weak increase with the freestream Mach number. Both the current DNS and the experimental measurements by Laufer ${ }^{1}$ show that the bulk convection speed in the freestream increases with freestream Mach number, while the value reported by Kendall ${ }^{50}$ at $M_{\infty}=4.5$ is relatively higher. As expected, all the freestream bulk convection speeds fall within the region where $M_{r}>1$, with $M_{r} \equiv\left(U_{\infty}-U_{b}\right) / a_{\infty}$.

Figures $12 \mathrm{a}$ and $12 \mathrm{~b}$ show that the instantaneous pressure field in the freestream exhibits a 'Mach-wave' radiation pattern with a preferred direction. The fact that the radiation wave front for the Mach 6 case is shallower than the Mach 2.5 case is consistent with the experimentally measured trend. ${ }^{1}$ By assuming that the acoustic radiation consists of plane waves generated by 'effective' acoustic sources within the boundary layer, the convection speed of acoustic sources, $U_{s}$, can be calculated. It is shown that the angle between the flow direction and plane-wave normal direction, $\theta_{n}$ can be determined by ${ }^{1}$

$$
\cos \theta_{n}=-\gamma M_{\infty} \frac{u^{\prime} / U_{\infty}}{p^{\prime} / p_{\infty}} \approx-\gamma M_{\infty} \frac{u_{r m s}^{\prime} / U_{\infty}}{p_{r m s}^{\prime} / p_{\infty}}
$$

where the negative sign specifies that the plane wave is radiating away from the boundary layer with $\theta_{n}>90^{\circ}$. Once $\theta_{n}$ or the wave-front orientation angle $\theta$ (shown in Fig. 12) is known, the velocity $U_{s}$ of sound sources that produce the waves can be determined by the 'Mach angle' relation

$$
\frac{1}{\sin \theta}=M_{r}=\frac{U_{\infty}-U_{s}}{a_{\infty}}
$$

By using the values of $u_{r m s}^{\prime} / U_{\infty}$ and $p_{r m s}^{\prime} / p_{\infty}$ listed in Table 2 for the Mach 6 case, Eq.(5) gives a value of $\theta_{n} \approx 120^{\circ}$ (correspondingly $\theta=30^{\circ}$ ), which agrees with the approximate inclination of the wave fronts from the numerical Schlieren image in Fig. 12a. Moreover, Eq. (6) gives the relative Mach number $M_{r}=2$ with the corresponding convection velocity of 'effective' radiation sources $U_{s} \approx 0.66 U_{\infty}$, which is slightly greater than the bulk convection speed $U_{b}=0.63$ calculated based on the space-time correlation (Fig. 11). An approximate agreement between the two convection speeds was also found for the Mach 2.5 case $^{21}$ with the 'effective' source speed $U_{s} \approx 0.4$ and $M_{r}=1.5$.

The supersonic value of relative Mach number for the 'effective' radiation sources as well as the match between the source convection velocity with the bulk convection speed $U_{b}$ supports the basic concept of 'eddy Mach wave ${ }^{45,46}$ and shows that the 'Mach wave type' radiation is produced by eddies which are convected supersonically with respect to the freestream. Although a quantitative validation of the theory is beyond the scope of this paper, the theory can be used to explain the observed variation in radiation intensity, directionality, and convection speed with freestreem Mach number. At low supersonic freestream Mach numbers, sources that contribute to the radiation field are primarily slowly moving ones, the convection velocities of which are supersonic relative to the freestream. As the Mach number increases, additional faster moving turbulent eddies acquire supersonic relative speeds and start to take part in the radiation process, acounting for the increased 'effective' source convection speed and enhanced radiation intensity. This explains the increase in $p_{r m s, \infty}^{\prime} / \tau_{w}$ from $M_{\infty}=2.5$ to $M_{\infty}=6$ as found in Fig. 4a. Moreover, the increased fraction of the inner layer that can radiate to the free stream contributes to a reduced gap between the peak frequency of fluctuations near the surface and within the free stream as shown by Fig. 7b. 


\section{Conclusions}

DNS is used to examine the pressure fluctuations generated by a hypersonic turbulent boundary layer with a nominal freestream Mach number of 6 . Detailed analyses of the statistical characteristics of pressure fluctuations, including the fluctuation intensities, frequency spectra, space-time correlations, and convection velocities, have been carried out. The DNS results are compared with the recently reported Mach $2.5 \mathrm{DNS}^{21}$ to highlight the variation of acoustic characteristics with Mach number. It is found that the Mach 6 DNS exhibits increased radiation intensity, enhanced energy content at high frequencies, shallower orientation of wave fronts with respect to the flow direction, and larger convection velocity relative to the Mach 2.5 case. These variations in the freestream pressure field with Mach number agree well with the experimentally measured trends ${ }^{1}$ and are consistent with the 'Mach wave radiation' concept. ${ }^{45,46}$

\section{Acknowledgments}

This work was performed as part of the Supersonic Project of NASA's Fundamental Aeronautics Program (FAP). The authors would like to thank Prof. Pino Martín of the University of Maryland for providing the original code which has been modified for the current study. Technical discussions with Robert Rubinstein and Elizabeth Lee-Rausch at NASA Langley Research Center are also acknowledged. The simulations have been conducted using the Pleiades Supercomputer of the NASA Advanced Supercomputing (NAS) Division.

\section{References}

${ }^{1}$ Laufer, J., "Some Statistical Properties of the Pressure Field Radiated by a Turbulent Boundary Layer," Physics of Fluids, Vol. 7, No. 8, 1964, pp. 1191-1197.

${ }^{2}$ Pate, S. R. and Schuller, C. J., "Radiated Aerodynamic Noise Effects on Boundary Layer Transition in Supersonic and Hypersonic Wind Tunnels," AIAA Journal, Vol. 7, No. 3, 1969, pp. 450-457.

${ }^{3}$ Pate, S. R., "Measurements and Correlations of Transition Reynolds Numbers on Sharp Slender Cones at High Speeds," AIAA Journal, Vol. 9, No. 6, 1971, pp. 1082-1090.

${ }^{4}$ Stainback, P. C., "Hypersonic Boundary-Layer Transition in the Presence of Wind Tunnel Noise," AIAA Journal, Vol. 9 , No. 12, 1971, pp. 2475-2476.

${ }^{5}$ Laderman, A. J., "Review of Wind-Tunnel Freestream Pressure Fluctuations," AIAA Journal, Vol. 4, No. 15, 1976, pp. 605-608.

${ }^{6}$ Stetson, K. F., "Nosetip Bluntness Effects on Cone Frustrum Boundary-Layer Transition in Hypersonic Flow," AIAA Paper 83-1763, 1983.

${ }^{7}$ Bushnell, D. M., "Notes on Initial Disturbance Fields for the Transition Problem," Instability and Transition, edited by M. Y. Hussaini and R. G. Voigt, Springer-Verlag, Berlin, Vol. 1, 1990, pp. 217-232.

${ }^{8}$ Beckwith, I. E. and Miller, C. G., "Aerothermodynamics and Transition in High-Speed Wind Tunnels at NASA Langley," Annu. Rev. Fluid Mech., Vol. 22, 1991, pp. 419-439.

${ }^{9}$ Donaldson, J. and Coulter, S., "A Review of Free-Stream Flow Fluctuation and Steady-State Flow Quality Measurements in the AEDC/VKF Supersonic Tunnel A and Hypersonic Tunnel B," AIAA Paper 95-6137, 1995.

${ }^{10}$ Chokani, N., Shipiyuk, A. N., Sidorenko, A. A., and McGinley, C. B., "Comparison Between a Hybrid Constant-Current Anemometer and Constant-Voltage Anemometer in Hypersonic Flow," AIAA Paper 2004-2248, 2004.

${ }^{11}$ Bounitch, A., Lewis, D. R., and Lafety, J. F., "Experimental Study of Second-Mode Instabilities on a 7-Degree Cone at Mach 6," AIAA Paper 2011-1200, 2011.

${ }^{12}$ Rufer, S. J. and Berridge, D. C., "Experimental Study of Second-Mode Instabilities on a 7-Degree Cone at Mach 6," AIAA Paper 2011-3877, 2011

${ }^{13}$ Masutti, M., Chazot, E., and Carbo, M., "Disturbance Level Characterization of a Hypersonic Blow-Down Facility," To appear in AIAA Journal, 2013.

${ }^{14}$ Schneider, S. P., "Effects of High-Speed Tunnel Noise on Laminar-Turbulent Transition," Journal of Spacecraft and Rockets, Vol. 38, No. 3, 2001, pp. 323-333.

${ }^{15}$ Fedorov, A. V., "Receptivity of a High-Speed Boundary Layer to Acoustic Disturbances," Journal of Fluid Mechanics, Vol. 491, 2003, pp. 101-129.

${ }^{16}$ Zhong, X. and Wang, X., "Direct Numerical Simulation on the Receptivity, Instability, and Transition of Hypersonic Boundary Layers," Annu. Rev. Fluid Mech., Vol. 44, 2012, pp. 527-561.

${ }^{17}$ Kistler, A. L. and Chen, W. S., "The Fluctuating Pressure Field in a Supersonic Turbulent Boundary Layer," Journal of Fluid Mechanics, Vol. 16, 1963, pp. 41-64.

${ }^{18}$ Maestrello, L., "Radiation from and Panel Response to a Supersonic Turbulent Boundary Layer," Journal of Sound Vibration, Vol. 10, No. 2, 1969, pp. 261-262.

${ }^{19}$ Beresh, S. J., Henfling, J. F., Spillers, R. W., and Pruett, B. O. M., "Fluctuating Wall Pressures Measured beneath a Supersonic Turbulent Boundary Layer," Physics of Fluids, Vol. 23, No. 7, 2011, 075110.

${ }^{20}$ Dolling, D. S. and Dussauge, J. P., "A survey of measurements and measuring techniques in rapidly distorted compressible turbulent boundary layers," AGARDograph, Vol. 315, 1989, pp. 1-18. 
${ }^{21}$ Duan, L., Choudhari, M., and Wu, M., "Numerical Study of Pressure Fluctuations Due to High-Speed Turbulent Boundary Layers," AIAA Paper 2012-3070, 2012.

${ }^{22}$ Martín, M., "DNS of Hypersonic Turbulent Boundary Layers. Part I: Initialization and Comparison with Experiments," Journal of Fluid Mechanics, Vol. 570, 2007, pp. 347-364.

${ }^{23}$ Duan, L., Beekman, I., and Martín, M. P., "Direct Numerical Simulation of Hypersonic Turbulent Boundary Layers. Part 3: Effect of Mach Number," Journal of Fluid Mechanics, Vol. 672, 2011, pp. 245-267.

${ }^{24}$ Duan, L., Beekman, I., and Martín, M. P., "Direct Numerical Simulation of Hypersonic Turbulent Boundary Layers. Part 2: Effect of Wall Temperature," Journal of Fluid Mechanics, Vol. 655, 2010, pp. 419-445.

${ }^{25}$ Duan, L. and Martín, M. P., "Direct Numerical Simulation of Hypersonic Turbulent Boundary Layers. Part 4: Effect of High Enthalpy," Journal of Fluid Mechanics, Vol. 684, 2011, pp. 25-59.

${ }^{26}$ Jiang, G. S. and Shu, C. W., "Efficient Implementation of Weighted ENO Schemes," Journal of Computational Physics, Vol. 126, No. 1, 1996, pp. 202-228.

${ }^{27}$ Taylor, E. M., Wu, M., and Martín, M. P., "Optimization of Nonlinear Error Sources for Weighted Non-Oscillatory Methods in Direct Numerical Simulations of Compressible Turbulence," Journal of Computational Physics, Vol. 223, No. 1, 2006, pp. 384-397.

${ }^{28}$ Williamson, J., "Low-Storage Runge-Kutta Schemes," Journal of Computational Physics, Vol. 35, No. 1, 1980 , pp. 48-56.

${ }^{29} \mathrm{Xu}$, S. and Martín, M. P., "Assessment of Inflow Boundary Conditions for Compressible Turbulent Boundary Layers," Physics of Fluids, Vol. 16, No. 7, 2004, pp. 2623-2639.

${ }^{30}$ Morgan, B., Larsson, J., Kawai, S., and Lele, S. K., "Improving Low-Frequency Characteristics of Recycling/Rescaling Inflow Turbulence Generation," AIAA Journal, Vol. 49, No. 3, 2011, pp. 582-597.

${ }^{31}$ Thompson, K. W., "Time Dependent Boundary Conditions for Hyperbolic Systems," Journal of Computational Physics, Vol. 68, No. 1, Jan. 1987, pp. 1-24.

${ }^{32}$ Welch, P. D., "The Use of Fast Fourier Transform for the Estimation of Power Spectra: A Method Based on Time Averaging Over Short, Modified Periodograms," IEEE Trans. Audio Electroacoustics, Vol. AU-15, 1967, pp. 70-73.

${ }^{33} \mathrm{Wu}, \mathrm{M}$. and Martín, M. P., "Direct numerical simulation of supersonic boundary layer over a compression ramp," AIAA Journal, Vol. 45, No. 4, 2007, pp. 879-889.

${ }^{34} \mathrm{Wu}, \mathrm{M}$. and Martín, M. P., "Analysis of shock motion in shockwave and turbulent boundary layer interaction using direct numerical simulation data," Journal of Fluid Mechanics, Vol. 594, 2008, pp. 71-83.

${ }^{35}$ Priebe, S. and Martín, M. P., "Low-frequency unsteadiness in shock wave-turbulent boundary layer interaction," Journal of Fluid Mechanics, Vol. 699, 2012, pp. 1-49.

${ }^{36}$ Kovasznay, L. S. G., "Turbulence in Supersonic Flow," Journal of Aeronautical Sciences, Vol. 20, 1953, pp. 657-674.

${ }^{37}$ Logan, P., "Modal Analysis of Hot-Wire Measurements in Supersonic Turbulence," AIAA Paper 88-423, 1988.

${ }^{38}$ Smits, A. J. and Dussauge, J. P., Turbulent Shear Layers in Supersonic Flow, American Institute of Physics, 2nd ed., 2006.

${ }^{39}$ Bies, D. W., "A Review of Flight and Wind Tunnel Measurements of Boundary Layer Pressure Fluctuations and Induced Structure Reponse," NASA CR-626, 1966.

${ }^{40}$ Guarini, S. E., Moser, R. D., Shariff, K., and Wray, A., "Direct Numerical Simulation of a Supersonic Turbulent Boundary Layer at Mach 2.5," Journal of Fluid Mechanics, Vol. 414, 2000, pp. 1-33.

${ }^{41}$ Spalart, P. R., "Direct Simulation of a Turbulent Boundary Layer up to Re $e_{\theta}=1410$," Journal of Fluid Mechanics, Vol. 187, 1988, pp. 61-98.

${ }^{42}$ Bernardini, M. and Pirozzoli, S., "Wall Pressure Fluctuations beneath Supersonic Turbulent Boundary Layers," Physics of Fluids, Vol. 23, No. 8, 2011, 085102.

${ }^{43}$ Blake, W. K., Mechanics of Flow-Induced Sound and Vibration, Academic Press, Orlando, Florida, 1986.

${ }^{44}$ Bradshaw, P., "Inactive Motion and Pressure Fluctuations in Turbulent Boundary Layers," Journal of Fluid Mechanics, Vol. 30, 1967, pp. 241-258.

${ }^{45}$ Phillips, O. M., "On the Generation of Sound by Supersonic Turbulent Shear Layers," Journal of Fluid Mechanics, Vol. 9, 1960, pp. 1-28.

${ }^{46}$ Williams, J. E. F. and Maidanik, G., "The Mach wave field radiated by supersonic turbulent shear flows," Journal of Fluid Mechanics, Vol. 21, 1965, pp. 641-657.

${ }^{47}$ Willmarth, W. W., "Wall Pressure Fluctuations beneath Turbulent Boundary Layers," Annual Review of Fluid Mechanics, Vol. 7, 1975, pp. 13-36.

${ }^{48}$ Choi, H. and Moin, P., "On the space-time characteristics of wall-pressure fluctuations," Physics of Fluids, Vol. 2, No. 8, 1990, pp. 1450-1460.

${ }^{49}$ Tsuji, Y., Fransson, J. H. M., Alferdsson, P. H., and Johansson, A. V., "Pressure statistics and their scaling in highReynolds-number turbulent boundary layers," Journal of Fluid Mechanics, Vol. 585, 2007, pp. 1-40.

${ }^{50}$ Kendall, J. M., "Supersonic Boundary Layer Transition Studies," Space Program Summary, Vol. 3, 1970 , pp. 43-47. 
Table 1. Boundary layer properties at the station selected for the analysis $\left(x_{a}=55 \delta_{i}\right)$ of the acoustic field for the current Mach 6 DNS and Mach 2.5 DNS. $^{21}$

$\begin{array}{lccccccccccc}M_{\infty} & U_{\infty}(\mathrm{m} / \mathrm{s}) & \rho_{\infty}\left(\mathrm{kg} / \mathrm{m}^{3}\right) & T_{\infty}(\mathrm{K}) & T_{w}(\mathrm{~K}) & R e_{\theta} & R e_{\tau} & R e_{\delta 2} & \theta(\mathrm{mm}) & \mathrm{H} & \delta(\mathrm{mm}) & \delta_{i}(\mathrm{~mm}) \\ 5.85 & 870.2 & 0.039 & 55.0 & 300.1 & 9659.2 & 464.2 & 1783.3 & 0.968 & 13.6 & 24.4 & 13.8 \\ 2.5 & 823.6 & 0.1 & 270.0 & 568.0 & 2834.8 & 509.9 & 1656.9 & 0.583 & 4.14 & 7.69 & 4.0\end{array}$

Table 2. The disturbance field in the freestream for the Mach 6 DNS at $z / \delta=3.2, R e_{\tau}=464$.

\begin{tabular}{rccccccc}
\hline$u_{r m s}^{\prime} / \bar{u}$ & $v_{r m s}^{\prime} / \bar{u}$ & $w_{r m s}^{\prime} / \bar{u}$ & $p_{r m s}^{\prime} / \bar{p}$ & $\rho_{r m s}^{\prime} / \bar{\rho}$ & $T_{r m s}^{\prime} / \bar{T}$ \\
$1.26 \times 10^{-3}$ & $0.99 \times 10^{-3}$ & $2.00 \times 10^{-3}$ & $2.06 \times 10^{-2}$ & $1.47 \times 10^{-2}$ & $5.86 \times 10^{-3}$ \\
& $(\rho u)_{r m s}^{\prime} / \overline{\rho u}$ & $T_{t, r m s}^{\prime} / \bar{T}_{t}$ & $p_{t, r m s}^{\prime} / \bar{p}_{t}$ & $\frac{\overline{\left(\partial u_{i} / \partial x_{i}\right)^{\prime 2}}}{\overline{\bar{\Omega}_{i}^{\prime} \Omega_{i}^{\prime}}}$ & $s_{r m s}^{\prime} / R$ \\
& $1.38 \times 10^{-2}$ & $1.76 \times 10^{-3}$ & $6.15 \times 10^{-3}$ & 1131.2 & $1.75 \times 10^{-4}$ \\
\hline
\end{tabular}

Table 3. The disturbance field in the freestream for the Mach 2.5 DNS at $z / \delta=2.8, \operatorname{Re}_{\tau}=510 .^{21}$

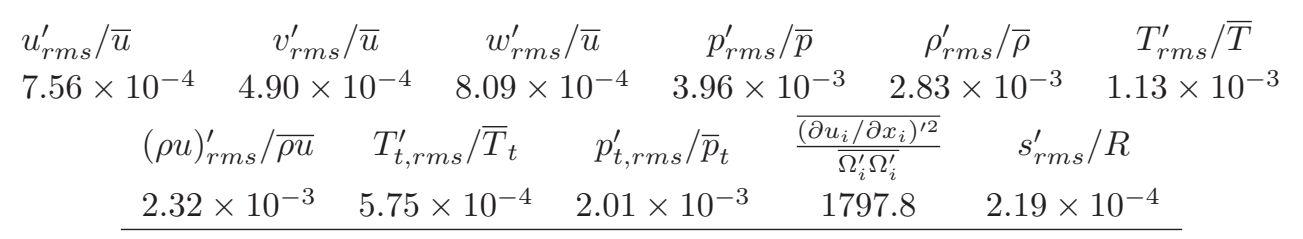




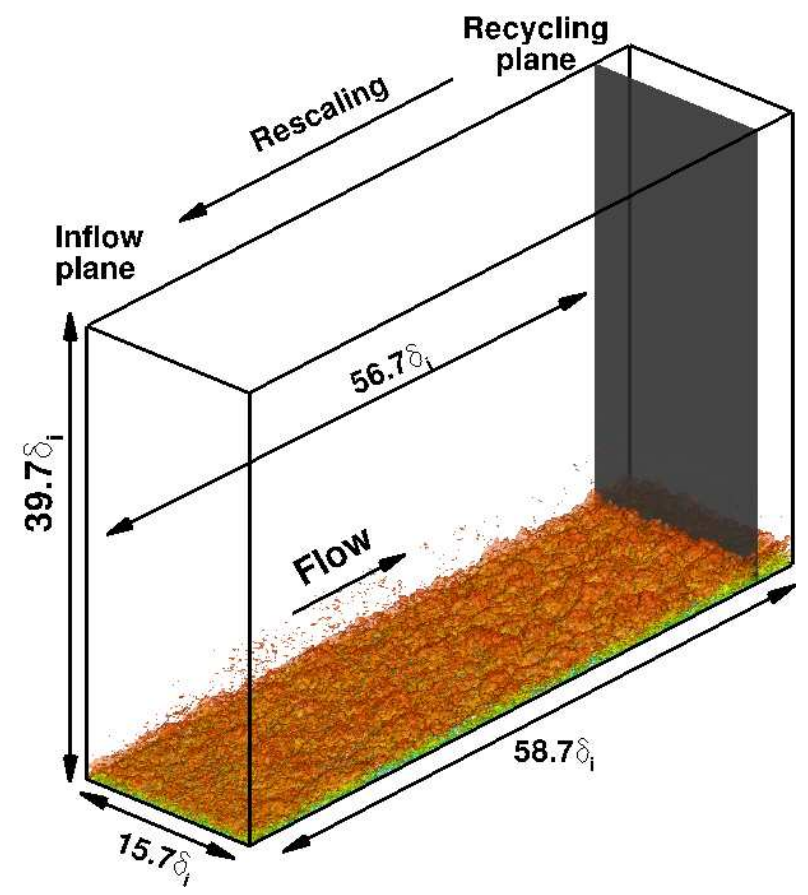

Figure 1. Computational domain and simulation setup. The reference length $\delta_{i}$ is the thickness of the boundary layer (based on $99 \%$ of the freestream velocity) at the inflow plane. An instantaneous flow field is shown in the domain, visualized by an iso-surface of the magnitude of density gradient, $|\nabla \rho| \delta_{i} / \rho_{\infty}=0.98$, colored by the streamwise velocity component (with levels from 0 to $U_{\infty}$, blue to red). Note that $x, y$, and $z$ are, respectively, the streamwise, spanwise, and wall-normal coordinates.

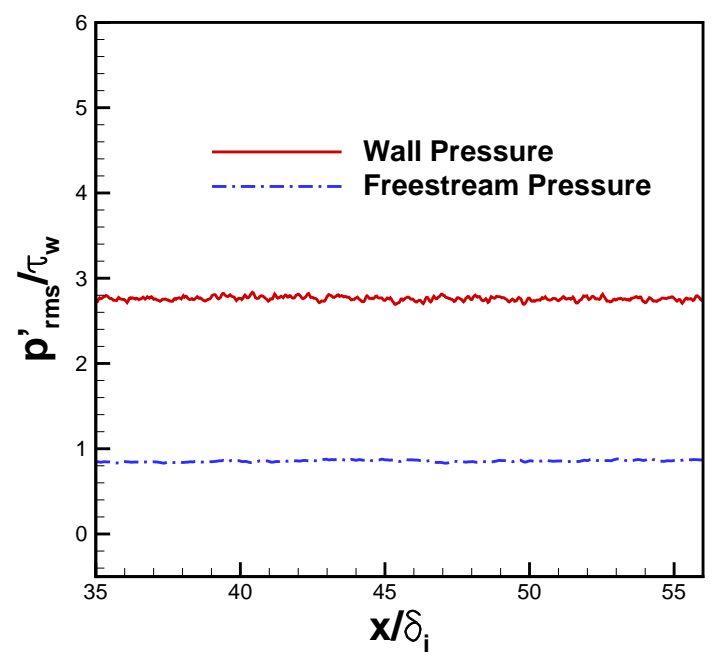

Figure 2. The streamwise distribution of the normalized pressure fluctuation rms at the wall $(z / \delta=0)$ and in the freestream $(z / \delta=3.2)$ for the Mach 6 DNS. 


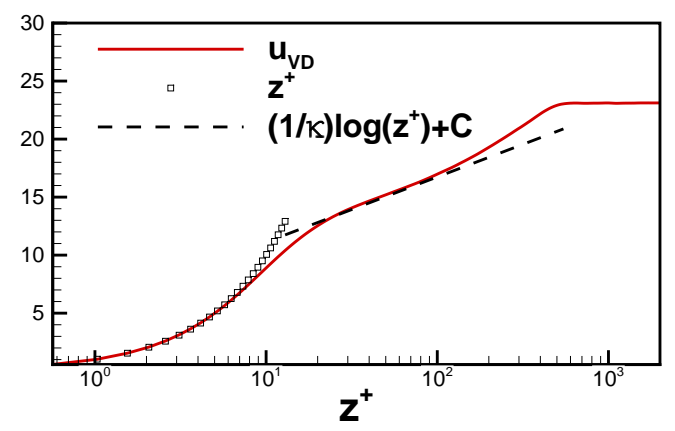

Figure 3. van Driest transformed mean velocity profile for the Mach 6 DNS $(\kappa=0.41, C=5.5)$.

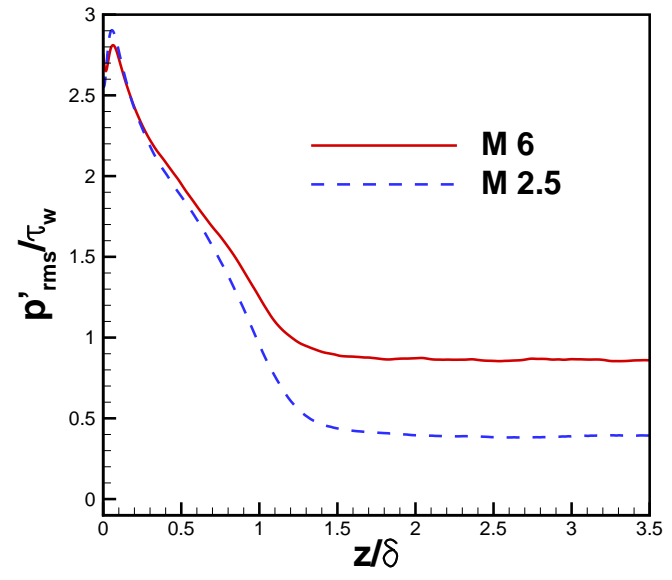

(a)

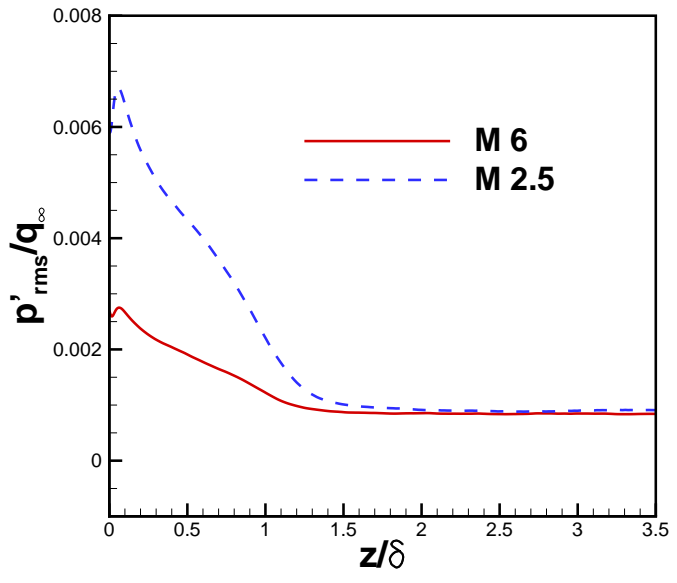

(b)

Figure 4. Pressure fluctuation rms profile for the Mach 6 and Mach 2.5 DNS. $^{21}$ (a) normalized by wall shear stress $\tau_{w}$; (b) normalized by freestream dynamic pressure $q_{\infty}$. 


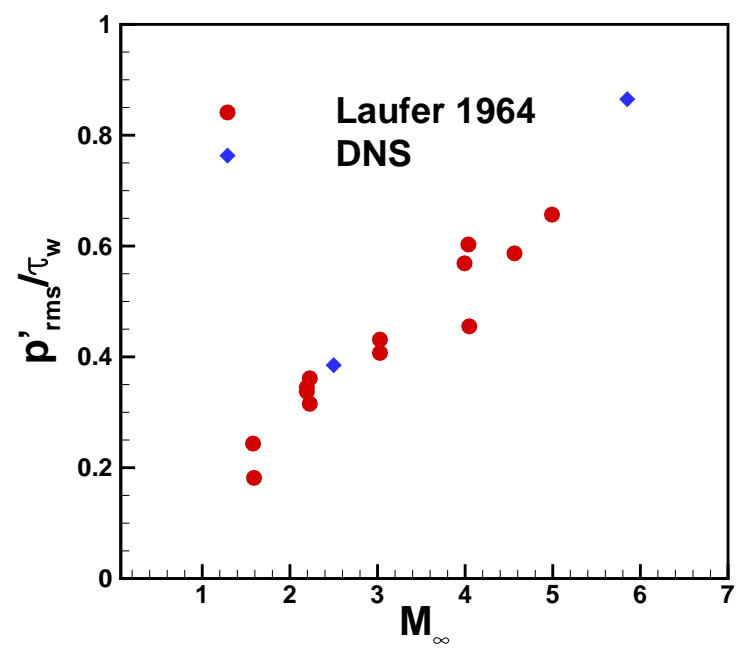

Figure 5. Intensity of freestream pressure fluctuation for the Mach 6 and Mach $2.5 \mathrm{DNS}^{21}$ com- $^{2}$ pared with the experiments by Laufer. ${ }^{1}$

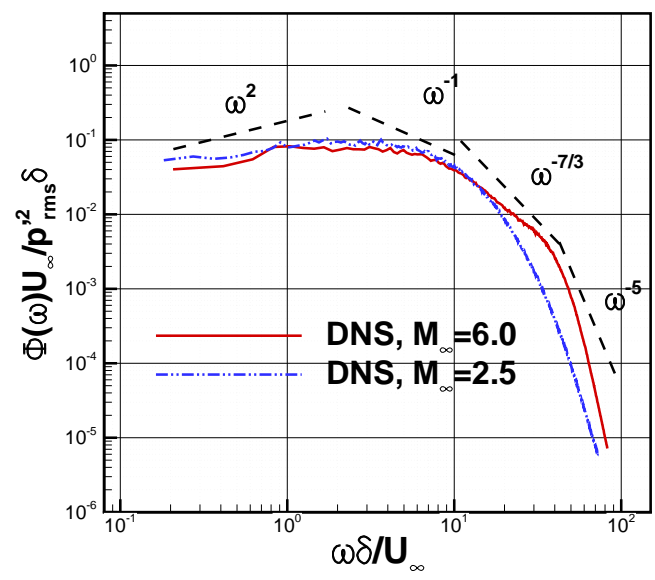

(a) Wall

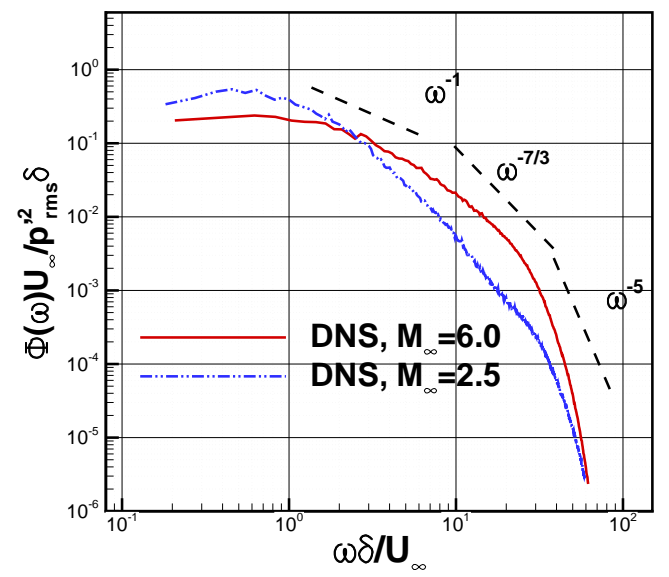

(b) Freestream

Figure 6. Normalized frequency spectrum of computed pressure signal at selected heights for the Mach 6 and Mach 2.5 DNS. $^{21}$ The freestream are taken at $z / \delta=3.2$ for the Mach 6 DNS and $z / \delta=2.8$ for the Mach 2.5. 


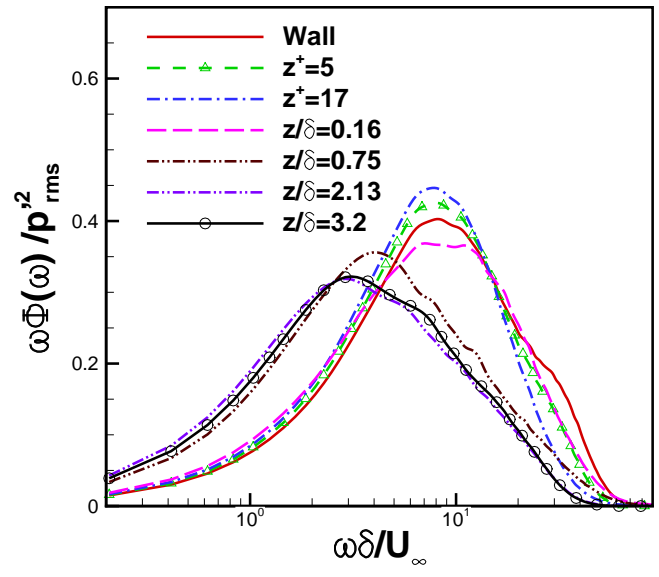

(a)

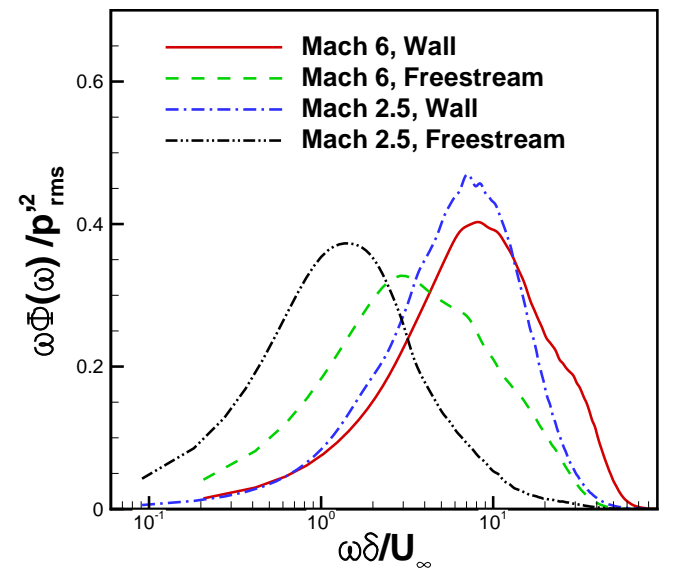

(b)

Figure 7. Pre-multiplied power spectrum of pressure signals. (a) at selected heights for the Mach 6 DNS; (b) comparison between Mach 6 and Mach 2.5. ${ }^{21}$ The pressure spectrum is normalized so that the area under each curve is equal to unity. The freestream are taken at $z / \delta=3.2$ for the Mach 6 DNS and $z / \delta=2.8$ for the Mach 2.5.

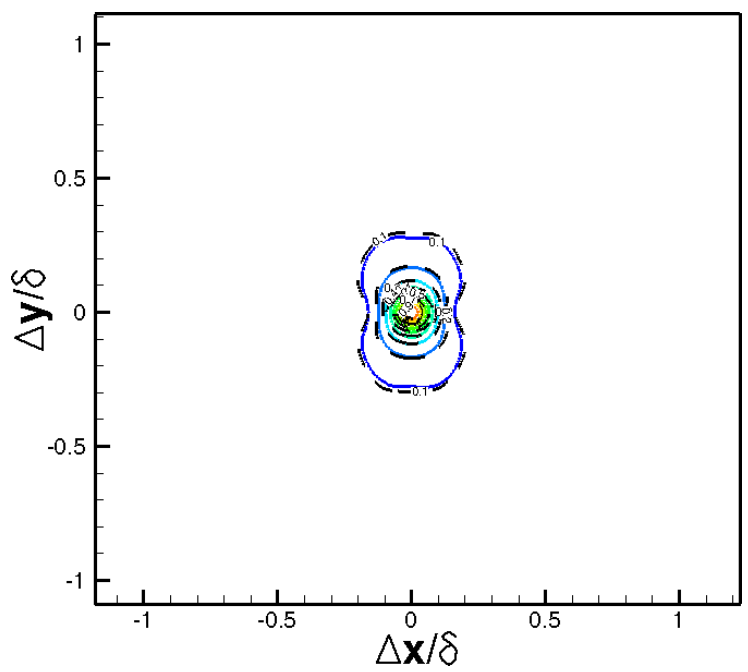

(a) Wall

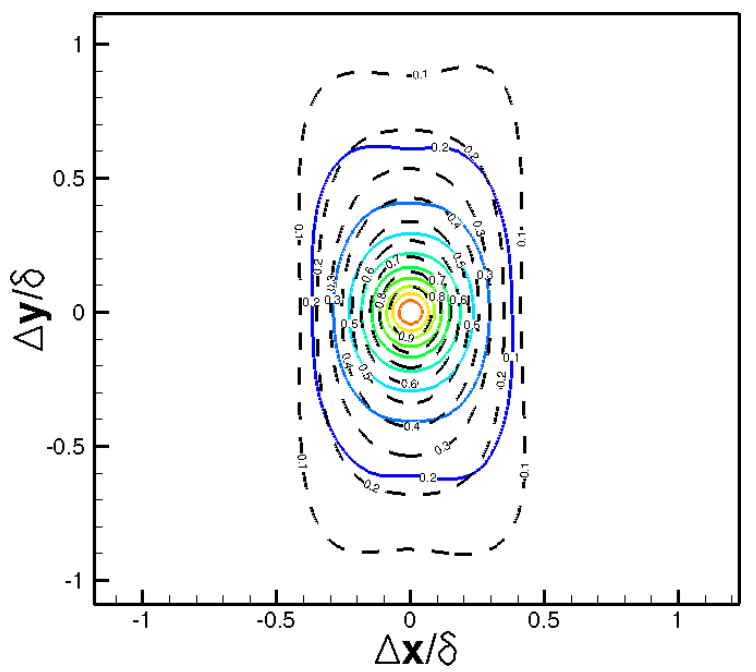

(b) Freestream

Figure 8. Streamwise-spanwise correlation coefficient of the pressure signal at selected heights for the Mach 6 DNS (Solid line) and Mach $2.5 \mathbf{D N S}^{21}$ (Dashed line). Contour levels vary from 0.1 to 0.9 with increments of 0.1 . The freestream are taken at $z / \delta=3.2$ for the Mach 6 DNS and $z / \delta=2.8$ for the Mach 2.5 . 


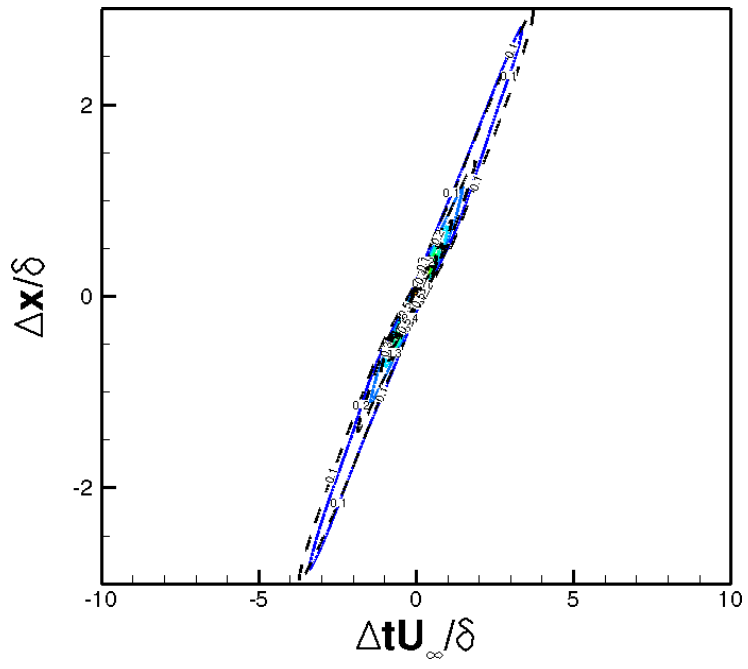

(a) Wall

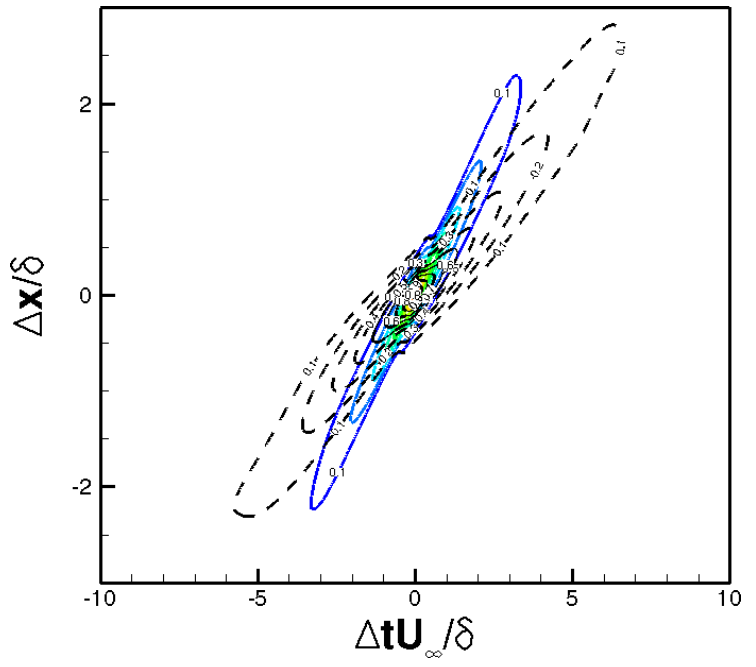

(b) Freestream

Figure 9. Space-time correlation coefficient of the pressure signal at selected heights for the Mach 6 DNS (Solid line) and Mach $2.5 \mathbf{D N S}^{21}$ (Dashed line). Contour levels vary from 0.1 to 0.9 with increments of 0.1 . The freestream are taken at $z / \delta=3.2$ for the Mach 6 DNS and $z / \delta=2.8$ for the Mach 2.5.

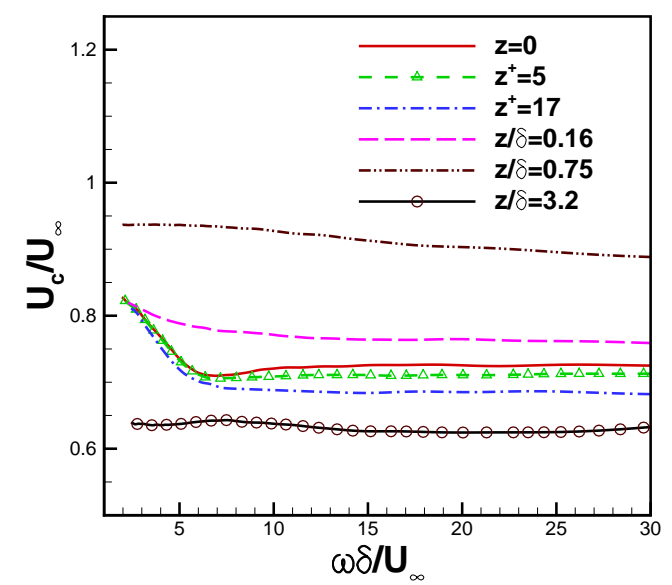

(a)

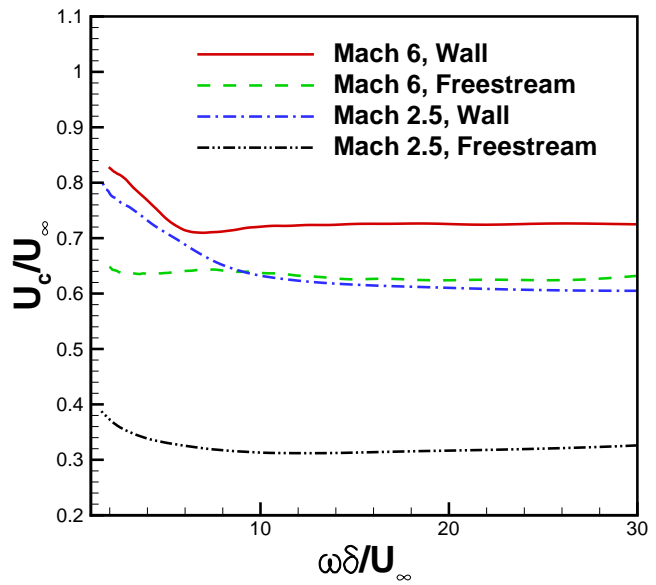

(b)

Figure 10. Convection speed of the pressure fluctuation as a function of frequency at selected heights. (a) at selected heights for the Mach 6 DNS; (b) comparison between Mach 6 and Mach $2.5 .^{21}$ The freestream are taken at $z / \delta=3.2$ for the Mach 6 DNS and $z / \delta=2.8$ for the Mach 2.5. 


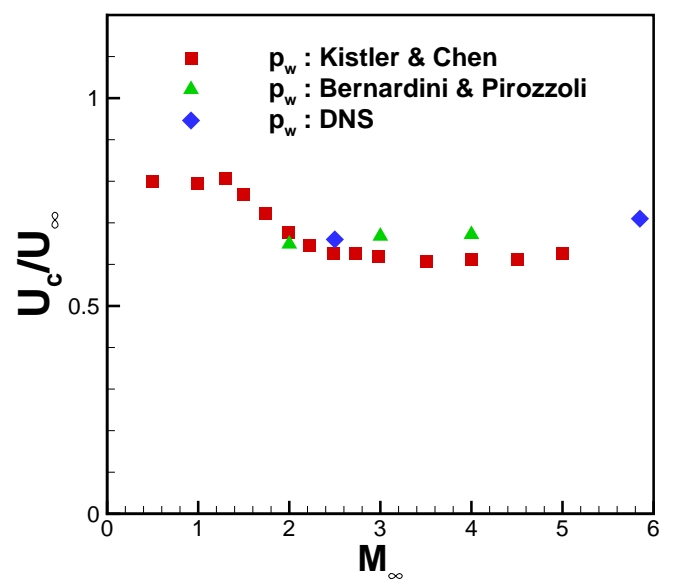

(a)

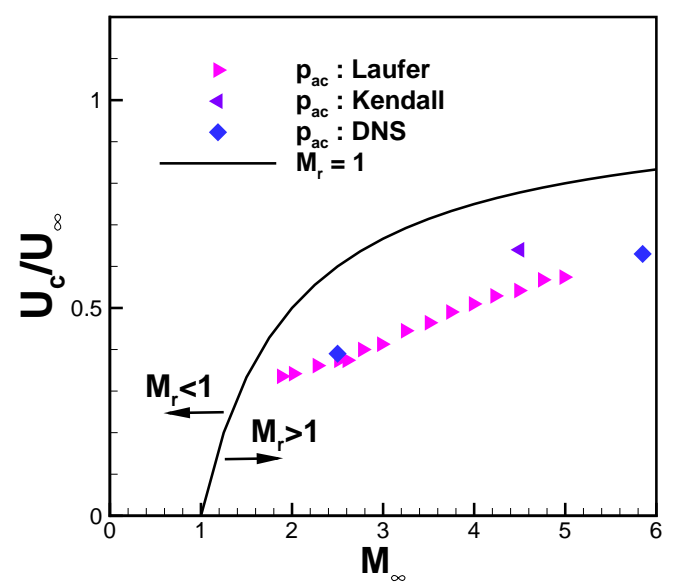

(b)

Figure 11. Bulk convection speeds of the pressure fluctuation as a function of freestream Mach number. (a) at the wall; (b) in the freestream. Symbols: squares, Kistler \& Chen; ${ }^{17}$ deltas, Bernardini \& Pirozzoli $;{ }^{42}$ right triangles, Laufer $;{ }^{1}$ left triangles, Kendall $;{ }^{50}$ diamonds, the present DNS and Mach 2.5 DNS. ${ }^{21}$ Lines: $M_{r}=1$.

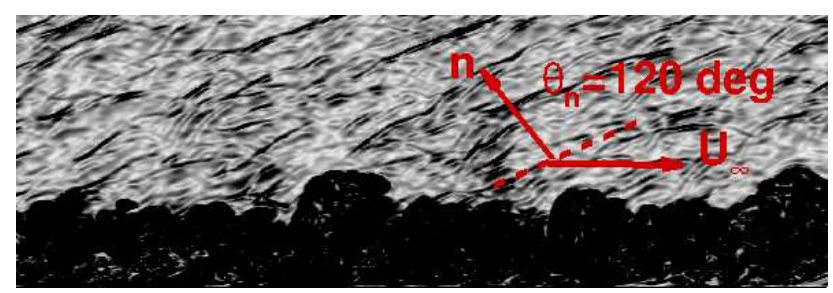

(a)

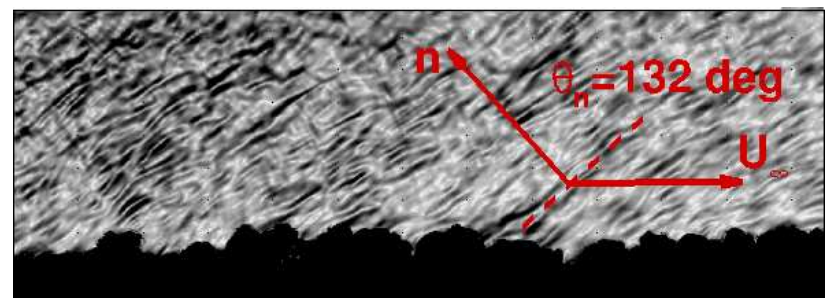

(b)

Figure 12. Numerical Schlieren image based on instantaneous flow field. (a) Mach 6 (b) Mach 2.5. ${ }^{21}$ Contour levels are selected to emphasize disturbances in the freestream. In (a), the dashdot line indicates the orientation of the radiation wave front; $n$ is the direction normal to the wave front; $U$ is the flow direction; $\theta_{n}$ is the angle between $U$ and $n$. 